\title{
Graphical Solution for the Back Pressurization Method of Hermetic Test
}

\author{
STANLEY RUTHBERG
}

\begin{abstract}
The back pressurization method for leak-testing hermetically sealed electronic packages requires gas-flow modeling to relate indicated leakage rates to true leak size. The molecular flow relationship which is appropriate for fine leak sizes is nonlinear and requires a numerical solution, which in actual test application may involve either many trial calculations or the use of approximations that lead to limiting case values. A new graphical procedure is presented for complete solution of the molecular flow equation for any given test condition and package volume through the use of a single set of characteristic curves and a test line. The effects of repetitive testing and of prefill with tracer gas are also considered. The characteristic curves are appropriate for both the helium leak detector and the radioisotope methods of test, while the form of the test line distinguishes between the two methods.
\end{abstract}

\section{INTRODUCTION}

$\mathbf{T}$ HE BACK pressurization method for leak-testing hermetically sealed packages is accomplished by forcing a tracer gas into the package interior and then measuring the quantity of gas that has penetrated the leak channel. The two tracer gases most commonly used for semiconductor devices are helium and radioactive krypton. With helium as the tracer gas, measurement is made by extracting the gas back through the leak into the helium mass spectrometer leak detector; with radioisotope $\mathrm{Kr}^{85}$ as the tracer, an external gamma counter determines the activity of the internal gas. Neither technique gives the true leak rate value directly; rather, both require a knowledge of the gas transport mechanism and the use of appropriate mathematical models for the determination of test parameters. As the gas transport mechanism for leaks under pressurization is that of transition flow [1], which combines the elements of molecular and laminar viscous flow in a manner not amenable to direct analytical solution [2], a number of simplifying flow models have been used.

Traditionally the molccular flow approximation which is relevant for very fine leaks has been used to relate the helium leak detector indication to the leak size, but even here a transcendental relationship is obtained which is doublevalued in leak size for each machine indication [3]. Thus numerical calculations are required to select pressurization parameters and to determine leak size. If precise values of leak size are required as in test evaluation or comparison, it is necessary either to use successive approximations or to construct a family of curves representing solutions for each parametric variation [4]. If an approximate correlation is sufficient, extensive tables of computer solutions for discrete

Manuscript received July 14, 1980; revised December 5, 1980

The author is with the Electron Devices Division, National Bureau of Standards, Washington, DC 20234. values of parameters have been available [5]. Finally, if only approximate values for the minimum detectable leak size and/or the maximum detectable leak size are sufficient, as for screening purposes, graphs are available for a limited range of parametric values [6].

The radioisotope test method is usually modeled by the laminar viscous flow approximation [7] which is relevant in principle to the larger leak sizes. The assumption is made that the internal gas pressure remains small and increases linearly with time under pressurization. The loss of gas back through the leak is also neglected [8]. While such modeling leads to a simple relationship between gamma-ray count rate and leak size, correlation of the results between this method and the helium leak-detector method is obscured.

In this paper a graphical procedure is presented for complete solution of the molecular flow equation for any given test condition. Only a single set of characteristic curves is required along with the use of a test line. The selection of parametric values for pressurization, time of pressurization, and lapsed time to achieve a given leak-test range for any package volume is readily made, and the effect on test results due to variation in parameters is easily visualized. The procedure is applied to both the helium and the radioisotope methods, and a comparison of operational behavior is derived for the fine leak range for which molecular flow is appropriate. In addition the case of repetitive testing is considered. Either prior testing or a prefill of packages with tracer gas can affect test results considerably, yet this situation has not been approached previously on a formal basis. Correction of results is particularly appropriate for test evaluation and interlaboratory comparisons.

\section{INTERNAL FRACTIONAL PARTIAL PRESSURE}

\section{A. Exact Solution}

The gas transport into and out of a package duc to a molecular flow leak is described by

$$
V \frac{d P}{d t}=F\left(P_{1}-P_{2}\right)
$$

where $V$ is the internal free volume of the package available to gas collection, $V d P / d t$ is the flow rate into (or out of) the package at ambient temperature, $F$ is the molecular-flow conductance of the leak channel [9], and $P_{1}$ and $P_{2}$ are the partial pressure of the tracer gas at each end of the leak channel. By definition the standard leak rate $L$ for a given gas is that flow rate obtained with $1 \mathrm{~atm}$ of gas pressure up- 
stream to the leak channel and zero pressure downstream so that from (1)

$$
L=F P_{0}
$$

for $P_{0}$ equal to $1 \mathrm{~atm}[10]$. It then follows from (1) and (2) that when a previously unexposed package has been immcrsed in a tracer gas at a pressure $P_{E}$ for a period $T$, the internal partial pressure $P$ is

$$
P=P_{E}\left[1-\exp \left(-\frac{L}{P_{0} V} T\right)\right] .
$$

When the pressurization ceases the tracer gas effuses back through the leak so that the interior tracer gas pressure decays exponentially. Then for any given lapsed or dwell time $t$

$$
P=P_{E}\left[1-\exp \left(-\frac{L}{P_{0} V} T\right)\right] \exp \left(-\frac{L}{P_{0} V} t\right) .
$$

If the interior partial pressure of the tracer gas is not zero but is initially $P^{\prime}$ due to a previous test or to a prefill of the package, the resultant pressure is

$$
\begin{aligned}
P= & \left\{P^{\prime}+\left[P_{E}-P^{\prime}\right]\left[1-\exp \left(-\frac{L}{P_{0} V} T\right)\right]\right\} \\
& \cdot \exp \left(-\frac{L}{P_{0} V} t\right) .
\end{aligned}
$$

The pressure change within the package is thus determined by a time constant

$$
\tau=\frac{P_{0} V}{L}
$$

or the relaxation rate $R_{r}$ which is the reciprocal of the time constant

$$
R_{r}=\frac{L}{P_{0} V} .
$$

Now the bracketed expression in (3) is a measure of the relative increase in the internal gas concentration, and this value normalized by $1 \mathrm{~atm}$ pressure is defined here as the quantity

$$
E=\frac{1}{P_{0}}\left[1-\exp \left(-R_{r} T\right)\right] \exp \left(-R_{r} t\right)
$$

which represents the internal fractional tracer gas pressure per atm of pressurization. This quantity $E$ will be used below to characterize the back pressurization method of hermetic test for both the helium leak detector and the radioisotope procedures.
A family of projections for $E$ as a function of the relaxation rate, with pressurization time and dwell time as parameters, is shown in Fig. 1 as derived from (6) for a range of parametric values sufficient for most semiconductor device packages; i.e., characteristics are included for a geometric series of values of pressurization time ranging from 0.1 to $300 \mathrm{~h}$ and values of dwell time ranging from $5 \mathrm{~min}$ to about $3 \mathrm{~h}$. The initial and final segments for these curves can be obtained readily from limiting solutions for small leaks and for large leaks as follows.

\section{B. Fine Leak Approximation}

For relatively small values of $R_{r}$, from (6)

$$
E \cong R_{r} T / P_{0} .
$$

Therefore with $T$ as a parameter the $E, R_{r}$ characteristics are initially linear projections at a $45^{\circ}$ slope on the log-log plane. A projection for any value of $T$ can be erected quickly by taking a coordinate point and passing a line of $45^{\circ}$ slope through the point, i.e., by calculating $E$ for a given $R_{r}$ or an $R_{r}$ for a given $E$ with $T$ as a parameter in (7). The locations of lines of intermediate values of $T$ are indicated in the figure by tick marks for $T=1-10$.

\section{Large Leak Approximation \\ For relatively large values of $R_{r}$$$
E \cong \frac{1}{P_{0}} \exp \left(-R_{r} t\right)
$$

or

$$
R_{r} \cong-\frac{1}{t} \ln \left(P_{0} E\right) .
$$

Thus the relaxation rate increases directly as the dwell time diminishes, the pressurization time is not a significant factor, and the relaxation rate changes little with large variation in $E$ for any given dwell time. This downslope segment for any $E(t)$ can be approximated readily by considering numerical values of $E$ of the form

$$
E=\frac{1}{P_{0}} \times 10^{-n}
$$

whence

$$
R_{r} \cong \frac{2.303 n}{t}
$$

and by joining with a straight line segment two points calculated from (9) for the selected value of $t$ and two successive values of $n$ in the $R_{r}$ region of interest.

\section{HELIUM LEAK DETECTOR METHOD}

\section{A. Test Equations}

When the tracer gas is helium, the flow rate of helium from the package interior back through the leak conductance and 


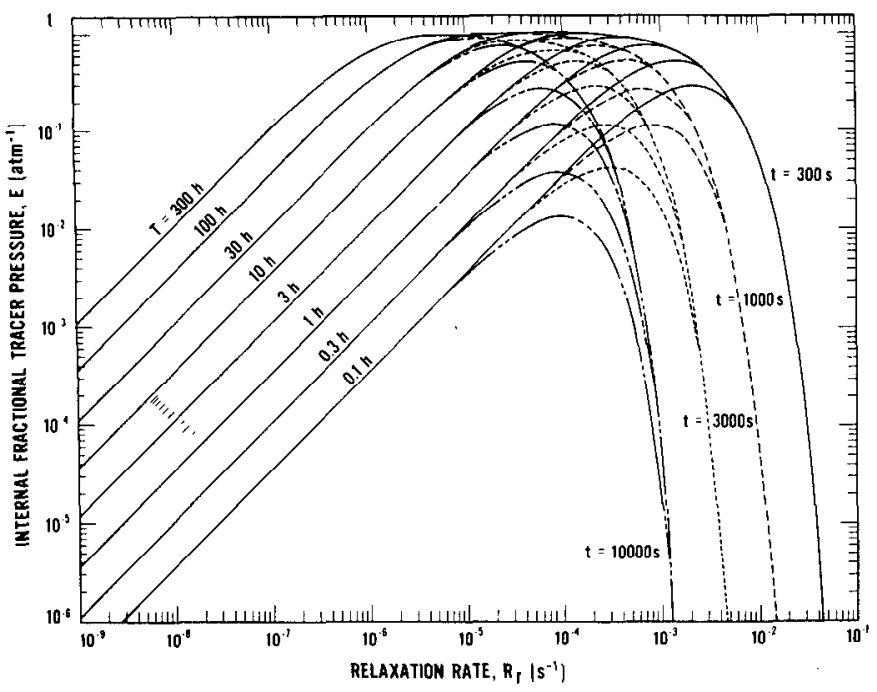

Fig. 1. E, the relative partial pressure of tracer gas within package interior free volume per atmosphere of external tracer gas pressurization, as function of relaxation rate $R_{r}$ with pressurization time $T$ and dwcll time $t$ as parameters. Transport mechanism is molecular flow.

into the helium leak detector will by (1) give rise to an indicated leak rate $R$ given by

$$
R=F \cdot P
$$

where the pressure within the leak detector is much less than $P$. Most tests for package leakage are done soon after assembly so that the initial interior helium concentration is zero. For such packages the leak detector indication is then from (2) and (3)

$$
R=P_{E} \cdot L \cdot E
$$

or from (5a)

$$
\frac{R}{P_{0} V P_{E}}=R_{r} \cdot E
$$

Thus the solution is characterized by the two factors $E$ and $R_{r}$, and all solutions relating leak detector response to standard leak rate may be derived from the characteristic projections for $E$ along with an appropriately placed test line.

\section{B. Test Lines}

It is apparent from (11) that the locus of all solutions for any test sequence will lie on a line of $45^{\circ}$ negative slope on the $\log E-\log R_{r}$ plane; for, with a given volume, indicated leak rate and pressurization the product $R_{r} \cdot E$ is a constant. Fig. 2 includes test lines ranging from $1 \times 10^{-5}(\mathrm{~atm} \cdot \mathrm{s})^{-1}$ to $1 \times 10^{-10}(\mathrm{~atm} \cdot \mathrm{s})^{-1} \cdot 1$ A test line for any other value can be constructed similarly as a line of $45^{\circ}$ negative slope through a coordinate point represented by $R_{r} \cdot E=$ constant.

1 Although the SI system of metric units is now preferred, present engineering practice uses units of atm. $\mathrm{cm}^{3} / \mathrm{s}$ for leak rate and $\mathrm{lbf} / \mathrm{in}^{2}$ for pressures near or greater than $1 \mathrm{~atm}$. Converstion factors are $1 \mathrm{~Pa}=$ $1.451 \times 10^{-4} \mathrm{lbf} / \mathrm{in}^{2}, 1 \mathrm{~Pa} \cdot \mathrm{m}^{3} / \mathrm{s}=9.869 \mathrm{~atm} \cdot \mathrm{cm}^{3} / \mathrm{s}$, and $1 \mathrm{~atm}=$ $1.01325 \times 10^{5} \mathrm{~Pa}$.

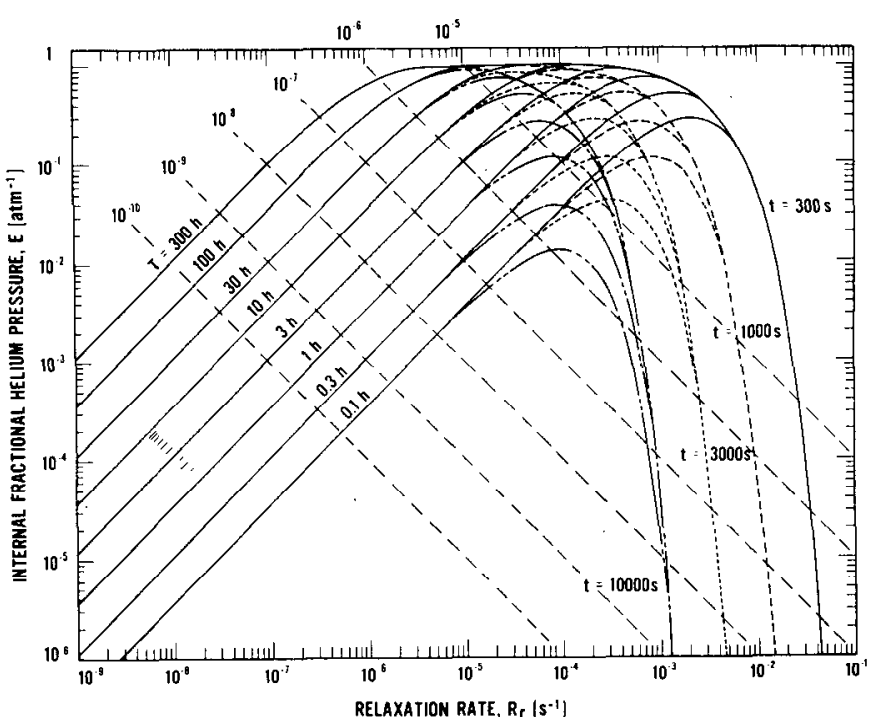

Fig. 2. Test lines for helium leak detector method. $E$ and $R_{r}$ are for helium gas. Test line values are for $R_{r} \cdot E=$ constant with indicated lines ranging from $10^{-5}$ to $10^{-10} \mathrm{~s}^{-1} \cdot \mathrm{atm}^{-1}$

Then specific solutions are defined by the intersection of the test line with that $E(T, t)$ characteristic corresponding to the particular test values of $T$ and $t$. Solutions to all modes of test operation may be so obtained.

1) Unknown Leak Size: In one mode of operation it may be desired to determine the leak size of a specimen of internal volume $V$ which has been subjected to an arbitrary set of back-pressurization parameters $P_{E}, T$, and $t$ for which an indicated leak rate $R$ was obtained. To solve this, the appropriate test line value is first computed from $R /\left(P_{0} \cdot V \cdot P_{E}\right)$. The intersection of this test line with the upslope portion $E(T)$ of the curve corresponding to the given pressurization time and with the downslope portion $E(t)$ corresponding to the given dwell time determine the two possible solutions for $R_{r}$ from which the standard leak rate for helium $L$ is determined. The leak size, which is defined as the standard leak rate for air, is $L / 2.69 .^{2}$

Example: Consider a volume of $0.1 \mathrm{~cm}^{3}$ pressurized to $5 \mathrm{~atm}$ abs ( $\sim 60 \mathrm{psig})$ in pure helium for $2 \mathrm{~h}$ and then put on the helium leak detector $50 \mathrm{~min}$ later to give a reading of $5 \times 10^{-8} \mathrm{~atm} \cdot \mathrm{cm}^{3} / \mathrm{s}$. The true leak size is required. The appropriate parameters follow.

$$
\begin{aligned}
V & =0.1 \mathrm{~cm}^{3} . \\
P_{E} & =5 \mathrm{~atm} \cdot \mathrm{abs} \\
T & =2 \mathrm{~h} . \\
t & =3000 \mathrm{~s} . \\
R & =5 \times 10^{-8} \mathrm{~atm} \cdot \mathrm{cm}^{3} / \mathrm{s} .
\end{aligned}
$$

The steps are as follows.

a) Compute $R_{r} \cdot E=R /\left(P_{0} \cdot V P_{E}\right)=1 \times 10^{-7}$ (atm s) ${ }^{-1}$.

b) In Fig. 2 find the intersection of the $1 \times 10^{-7}$ (atm . s) ${ }^{-1}$ test line with the $T=2 \mathrm{~h}$ characteristic. Since $T=2 \mathrm{~h}$ is not shown in Fig. 2, lay a scale parallel to

2 Leak rate for helium/leak rate for air $=$ (molecular weight air molecular weight helium) $1 / 2=2.69$, where $M^{\text {air }}=28.98[11]$. 
the $R_{r}, E$ characteristics and through the $2 \cdot h$ tick mark. Determine the value of $R_{r}$ at the intersection. $R_{r} \cong$ $3.6 \times 10^{-6} \mathrm{~s}^{-1}$. Therefore by $(5 \mathrm{a}) L$ the standard leak rate for helium is $3.6 \times 10^{-7} \mathrm{~atm} \cdot \mathrm{cm}^{3} / \mathrm{s}$ from which the leak size is $1.3 \times 10^{-7} \mathrm{~atm} \cdot \mathrm{cm}^{3} / \mathrm{s}$.

c) Follow the $1 \times 10^{-7}$ test line to the $t=3000 \mathrm{~s} E(t)$ characteristic. Determine the value of $R_{r}$ at the intersect. $R_{r}=2.8 \times 10^{-3} \mathrm{~s}^{-1}$. Therefore $L=2.8 \times 10^{-4}$ $\mathrm{atm} \cdot \mathrm{cm}^{3} / \mathrm{s}$ and $L_{\text {air }}=1 \times 10^{-4} \mathrm{~atm} \cdot \mathrm{cm}^{3} / \mathrm{s}$.

d) The leak size is either $1.3 \times 10^{-7}$ or $1 \times 10^{-4} \mathrm{~atm} \cdot$ $\mathrm{cm}^{3} / \mathrm{s}$. Discrimination is made with a followup reading on the leak detector at a later time. Any significant change indicates that the leak rate is the larger value.

2) Pressurization Parameters: By far most hermetic test activity is for screening purposes. This requires a selection of parametric values so that all specimens with leak sizes greater than some specified value be rejected. The upper limit of the test is determined, however, by the shortest dwell time that can be obtained, and it is hoped the test range can be made broad enough to overlap that of the gross leak test that would follow.

First the reject level relaxation rate is calculated from (5a) from the package-free volume and the specified standard leak rate for helium, and a vertical line is erected on the chart at that value. The test line is calculated from the left side of (11) with the selected value of leak detector signal $R$, the package volume $V$, and a convenient pressurization value $P_{E}$. The intersection of this test line with the $R_{r}$ line sets the choice for $T$. If the first test line selected produces an inconvenient value for $T$ one can move away from the first intersection to choose more satisfactory values for both $T$ and a test line. The only constraint is that the leak detector reject signal has to be greater than the minimum detectable signal by a suitable amount [12]. Then the corresponding maximum value of $R_{r}$ that can be detected is easily picked off by extending the test line to the $E(t)$ characteristic corresponding to the attainable dwell time. Alternatively the major factor in the test may be the maximum detectable leak size. Then the maximum value of $R_{r}$ is calculated and a vertical line extended from this value. The intersection with a suitable $E(t)$ characteristic determines the lower end of the test line which is then extended at a $-45^{\circ}$ slope to a suitable $E(T)$ characteristic.

Example: Consider an IC package of $0.01 \mathrm{~cm}^{3}$ nominal free volume for which a reject leak size of $5 \times 10^{-8} \mathrm{~atm}$. $\mathrm{cm}^{3} / \mathrm{s}$ of air is required [8]. Suppose the background signal is found to be $1 \times 10^{-9} \mathrm{~atm} \cdot \mathrm{cm}^{3} / \mathrm{s}$ of helium and a signalto-background value of five is selected. Also the tests could be completed within $50 \mathrm{~min}$ after release from the pressurization chamber. Consider $P_{E}=5 \mathrm{~atm} \cdot$ abs. The appropriate parameters follow:

$$
\begin{array}{ll}
V & =0.01 \mathrm{~cm}^{3}, \\
L_{\text {air reject }} & =5 \times 10^{-8} \mathrm{~atm} \cdot \mathrm{cm}^{3} / \mathrm{s}, \\
R_{\text {reject }} & =5 \times 10^{-9} \mathrm{~atm} \cdot \mathrm{cm}^{3} / \mathrm{s} \text { (helium), } \\
t & =3000 \mathrm{~s}, \\
P_{E} & =5 \mathrm{~atm} \cdot \text { abs. }
\end{array}
$$

Then

$$
\begin{array}{ll}
L & =1.35 \times 10^{-7} \mathrm{~atm} \cdot \mathrm{cm}^{3} / \mathrm{s}, \\
R_{r} & =1.35 \times 10^{-5} \mathrm{~s}^{-1}, \\
R_{\text {reject }} / P_{0} V P_{E} & =1 \times 10^{-7} \mathrm{~atm}^{-1} \cdot \mathrm{s}^{-1} .
\end{array}
$$

The intersection of test line $1 \times 10^{-7}$ and $R_{r}$ of $1.35 \times$ $10^{-5}$ is at $T=0.15 \mathrm{~h}$ (see Fig. 3). But $T=9 \mathrm{~min}$ is too short for convenience. Consider $T=0.3 \mathrm{~h}$ instead. Doubling the value of $T$ at constant $R_{r}$ doubles $E$, as seen in (7), and hence the value of the test line; thus the detector reject level is increased to $1 \times 10^{-8} \mathrm{~atm} \cdot \mathrm{cm}^{3} / \mathrm{s} . \mathrm{A}-45^{\circ}$ test line established at $T=0.3 \mathrm{~h}$ and $R_{r}=1.35 \times 10^{-5} \mathrm{~s}^{-1}\left(R_{r} E=2 \times\right.$ $10^{-7}$ ) intersects the $t=3000 \mathrm{~s}$ characteristic at $R_{r}=3.3 \times$ $10^{-3}$ so that the maximum standard leak rate for helium $L=3.3 \times 10^{-5} \mathrm{~atm} \cdot \mathrm{cm}^{3} / \mathrm{s}$ and then $L_{\mathrm{air}}=1.2 \times 10^{-5}$ $\mathrm{atm} \cdot \mathrm{cm}^{3} / \mathrm{s}$. However $1.2 \times 10^{-5}$ is borderline for any gross leak test, and the only way to increase the present limit significantly is to decrease the dwell time. Decreasing dwell time to the 1000-s ( 17-min) characteristic would lead to an $R_{r}$ of $1.1 \times 10^{-2}$ and hence an $L_{\text {air }} \sim 4.1 \times 10^{-5} \mathrm{~atm} \cdot$ $\mathrm{cm}^{3} / \mathrm{s}$ for some improvement in overlap between the fine and gross leak test. Note that an increase in dwell time beyond $\sim 1 \mathrm{~h}$ would pull the intersection point off the straight line region of $E(T)$. It should also be noted that $P_{E}$ could be dropped to $2.5 \mathrm{~atm}$ pressure and the original value of leak detector reject limit of $5 \times 10^{-9} \mathrm{~atm} \cdot \mathrm{cm}^{3} / \mathrm{s}$ be retained without changing the pressurization time test limits. Only the ratio of signal to background would be affected.

Since a leak size of $5 \times 10^{-8} \mathrm{~atm} \cdot \mathrm{cm}^{3} / \mathrm{s}$ in a $0.01 \cdot \mathrm{cm}^{3}$ volume produces a time constant of only 2 days, consider a decrease in the reject leak size by a factor of 5 to give $1 \times$ $10^{-8} \mathrm{~atm} \cdot \mathrm{cm}^{3} / \mathrm{s}$. Then $R_{r}$ becomes $2.7 \times 10^{-6} \mathrm{~s}^{-1}$ and the intersection of this value with the $2 \times 10^{-7}$ test line shows $T=7.5 \mathrm{~h}$, a 25 -fold increase in pressurization time; i.e., with (7) $R_{r} \cdot E \sim R_{r}^{2} T / P_{0}$.

3) Repetitive Measurement: Often the package may include an initial partial pressure of helium because of prior testing or prefill, and this initial concentration will affect the test results. In practice specimens are conditioned in vacuum or allowed to stand for some time until an initial leak detector reading is relatively small, but when such a long wait time may not be suitable or where some accuracy is required a formal correction procedure is desirable.

The specimen is first placed in the inlet of the leak detector and an initial measurement is made of the effusion. The effusion rate $R_{1}$ is related to the interior partial pressure $P_{1}$ and to the standard leak rate through (2) and (10) as

$$
R_{1}=P_{1} \frac{L}{P_{0}}
$$

After another delay period of $t^{\prime \prime}$, the specimen is subjected to hermetic test, and a final measurement is made. The initial partial pressure $P^{\prime}$ just prior to pressurization, after further possible effusion during the delay, is

$$
P^{\prime}=\frac{R_{1} P_{0}}{L} \cdot \exp \left(-R_{r} t^{\prime \prime}\right)
$$




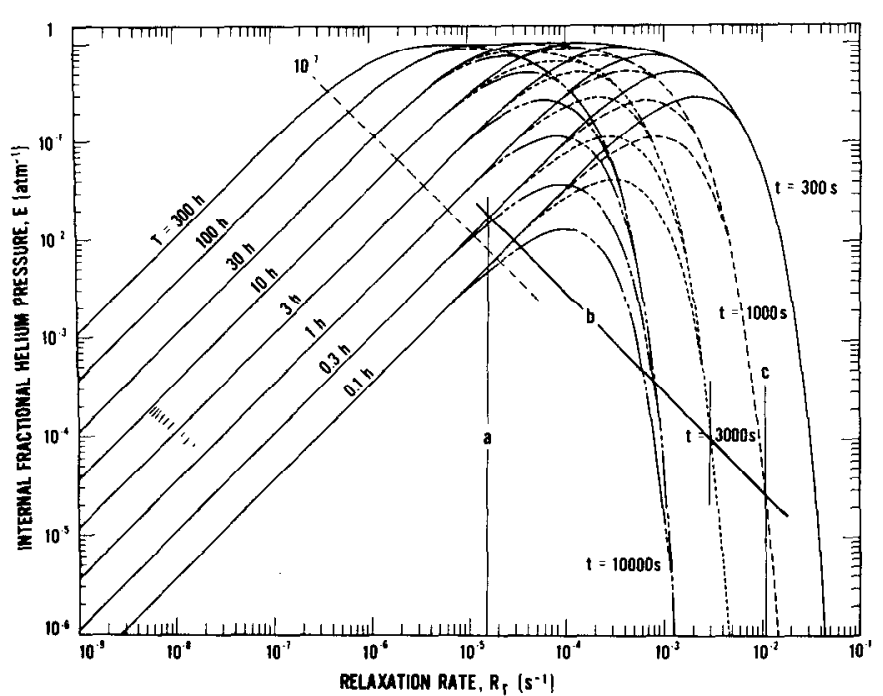

Fig. 3. Selection of pressurization parameters for helium leak detector method: an example. Reject leak size (air) of $5 \times 10^{-8} \mathrm{~atm} \cdot \mathrm{cm}^{3} / \mathrm{s}$ with $V=0.01 \mathrm{~cm}^{3}, P_{E}=5 \mathrm{~atm}, t=3000 \mathrm{~s}$, and minimum detectable signal of $5 \times 10^{-9} \mathrm{~atm} \cdot \mathrm{cm}^{3} / \mathrm{s}$ for signal-to-noise of 5 . Line (a) represents an $R_{r}$ of $1.35 \times 10^{-5} \mathrm{~s}^{-1}$ for helium. Then $R /\left(P_{0} V P_{E}\right)=1 \times$ $10^{-7} \mathrm{~s}^{-1} \cdot \mathrm{atm}^{-1}$. First intersection of 10-7 test line and (a) at $T \sim 0.15 \mathrm{~h}$ is not satisfactory, so $T=0.3 \mathrm{~h}$ is chosen. Line (b) becomes the test line. Intersection of (b) at $t=3000 \mathrm{~s}$ leads to leak size of $1.2 \times 10^{-5} \mathrm{~atm} \cdot \mathrm{cm}^{3} / \mathrm{s}$ of air. Line (c) for intersect at $t=$ $1000 \mathrm{~s}$ leads to $L_{\text {air }} \approx 4.1 \times 10^{-5} \mathrm{~atm} \cdot \mathrm{cm}^{3} / \mathrm{s}$.

The measured leak rate $R_{2}$ obtained after pressurization is equal to $L P / P_{0}$ where $P$ is now described by (4). With (4), (6), and (12), it follows that

$$
\begin{aligned}
R_{2}= & R_{1} \exp \left(-R_{r} t^{\prime \prime}\right)\left[\exp \left(-R_{r} t_{2}\right)-P_{0} E_{2}\right] \\
& +P_{0} V P_{E} R_{r} E_{2}
\end{aligned}
$$

where the subscript 2 denotes the quantities related to the pressurization sequence. As a general approach, the solution is obtained by successive approximation as follows.

a) Compute an approximate test line value from

$$
\frac{R_{2}}{P_{0} V P_{E}} \cong R_{r} \cdot E_{2}
$$

b) With this test line find the approximate values for $R_{r}$ and $E_{2}$ at $E\left(T_{2}, t_{2}\right)$, here designated as $R_{r}{ }^{\prime}$ and $E_{2}{ }^{\prime}$.

c) Compute the value of $\exp \left(-R_{r}{ }^{\prime} t_{2}\right)$ from the value of $t_{2}$ used in the test.

d) Compute the value of $\exp \left(-R_{r} t^{\prime \prime}\right)$ from the measured value of $t^{\prime \prime}$.

e) With these approximate values from $b, c, d$

$$
\frac{R_{2}-R_{1} \exp \left(-R_{r}^{\prime} t^{\prime \prime}\right)\left[\exp \left(-R_{r}^{\prime} t_{2}\right)-P_{0} E_{2}^{\prime}\right]}{P_{0} V P_{E}} \cong R_{r} \cdot E_{2} .
$$

This then is a corrected test line from which the two possible solutions may be obtained at the intersects for $E\left(T_{2}\right)$ and $E\left(t_{2}\right)$. Further iteration can be made.
If however the first approximated test line crosses the $E(T)$ characteristics in the region of straight line projections, then

$$
\begin{aligned}
& \exp \left(-R_{r} t^{\prime \prime}\right) \rightarrow 1 \\
& \exp \left(-R_{r} t_{2}\right) \rightarrow 1 \\
& E_{2} \ll 1,
\end{aligned}
$$

so that by (14)

$$
\frac{R_{2}-R_{1}}{P_{0} \cdot V \cdot P_{E}}=R_{r} \cdot E_{2}
$$

as one might expect.

At the large leak end of the test line, the solution is somewhat insensitive to variation in test line value since $R_{r}$ is primarily a function of $t_{2}$, i.e.,

$$
\begin{aligned}
& \exp \left(-R_{r} t^{\prime \prime}\right)<1 \\
& \exp \left(-R_{r} t_{2}\right) \rightarrow 0 \\
& E_{2} \ll 1
\end{aligned}
$$

so that

$$
\frac{R_{2}}{P_{0} V P_{E}} \cong R_{r} \cdot E_{2}
$$

and the prereading does not produce a significant correction.

Thus one only need see where the approximated test line falls to determine how much further iteration is required. If it falls in the fine leak area of straight line projections, simply take the difference between the final leak detector value and the prereading and calculate the test line value. The "true" value for $R_{r}$ is either at $E\left(T_{2}\right)$ or $E\left(t_{2}\right)$. If only the large leak value is desired, simply calculate the test line from $R_{2}$. If the approximated test line falls in the intermediate $R_{r}$ range, the iteration procedure is appropriate.

\section{RADIOISOTOPE METHOD}

\section{A. Test Equation}

Since the $\sim 0.5-\mathrm{MeV}$ gamma radiation cmitted by $\mathrm{Kr}^{85}$ easily penetrates most semiconductor package walls the amount of pure $\mathrm{K}_{\mathrm{I}}{ }^{85}$ that has passed through a leak channel and remains within the package cavity after pressurization in a $\mathrm{Kr}^{85}-\mathrm{N}_{2}$ gas mixture can be measured without resorting to extraction back through the leak channel as in the use of the helium leak detector. Thus the gamma count rate $R^{*}$ becomes

$$
R^{*}=(P V) \cdot A \cdot K
$$

where $P$ is the internal partial pressure of $\mathrm{Kr}^{85}, P V$ is the quantity of $\mathrm{Kr}^{85}$ at ambient temperature within the cavity, 
$A$ is the activity of pure $\mathrm{Kr}^{85}\left(\mu \mathrm{Ci} / \mathrm{atm} \cdot \mathrm{cm}^{3}\right),{ }^{3}$ and $K$ is the overall counting efficiency of the detector for the particular package type at a particular location within the crystal detector well (count rate $/ \mu \mathrm{Ci}$ ). The interior partial pressure is given by (3), but the external partial pressure $P_{E}$ is

$$
P_{E}=\frac{S}{A} \cdot P_{E}^{*}
$$

where $S$ is the specific activity of the $\mathrm{Kr}^{85}-\mathrm{N}_{2}$ gas mixture $\left(\mu \mathrm{Ci} / \mathrm{atm} \cdot \mathrm{cm}^{3}\right)$ and $P_{E}{ }^{*}$ is the pressurization value for the gas mixture. Thus

$$
\frac{R^{*}}{P_{0} \cdot V \cdot P_{E}^{*}}=S K \cdot E^{*}
$$

where the solution is characterized only by the internal fractional partial pressure for $\mathrm{Kr}^{85}$, here designated as $E^{*}$.

1) Fine Leak Approximation: For relatively small values of relaxation rate with krypton $R_{r}^{*}$ by (7) and (19),

$$
\frac{R^{*}}{P_{0} V \cdot P_{E}{ }^{*} \cdot S K} \cong \frac{R_{r}{ }^{*} T^{*}}{P_{0}},
$$

with $T^{*}$ denoting pressurization time with krypton. Thus

$$
\frac{R^{*} P_{0}}{P_{E}{ }^{*} K K T^{*}}=L^{*}
$$

where $L^{*}$ is the standard leak rate for $\mathrm{Kr}^{85}$. Package volume is not a factor, and (20) differs from the traditional recipe [7], [8] in that $P_{E}{ }^{*}$ is now raised only to the first power rather than to the second power as in the laminar viscous flow model.

\section{B. Test Lines}

For a given volume, indicated count rate, and pressurization in a particular gas mixture the locus of all solutions is now on a horizontal line of value $E^{*}$ rather than the $45^{\circ}$ slope of the helium leak detector, and there is now no need to construct a set of such lines superimposed on the $E, R_{r}$ plane. Specific solutions for the test sequence are as before at the intersection of the test line with the $E(T, t)$ characteristic specified by the parametric values of $T$ and $t$.

1) Pressurization Parameters-Example: Consider again the $0.01-\mathrm{cm}^{3}$ package to be leak tested to $5 \times 10^{-8} \mathrm{~atm} \cdot \mathrm{cm}^{3} / \mathrm{s}$ of air with a dwell time of $1000 \mathrm{~s}$. Background count rates are generally of the order of 500 counts/min, a typical value for $S$ is $200 \mu \mathrm{Ci} / \mathrm{atm} \cdot \mathrm{cm}^{3}$, and a typical value of $K$ is $10^{4}$ $\min ^{-1} \cdot \mu \mathrm{Ci}^{-1}$. Assume a signal-to-background ratio of 5 for the reject level count rate and a 5 -atm pressurization. It is desired to establish the required value of $T$ and the maximum detectable leak size $L_{\mathbf{a i r}}$. The appropriate parameters follow.

3 Although the SI system of metric units is now preferred, present engineering practice uses units of curie for disintegration rate; $1 \mathrm{Ci}=$ $3.7 \times 10^{10} \mathrm{~Bq}$ (events/s).

$$
\begin{array}{ll}
V & =0.01 \mathrm{~cm}^{3}, \\
L_{\mathrm{a}} \text { reject } & =5 \times 10^{-8} \mathrm{~atm} \cdot \mathrm{cm}^{3} / \mathrm{s} \\
R_{0}{ }^{*} & =500 \mathrm{~min}^{-1} \\
R^{*} \text { reject } & =2500 \mathrm{~min}^{-1} \\
S & =200 \mu \mathrm{Ci} / \mathrm{atm} \cdot \mathrm{cm}^{3} \\
K & =10^{4} \mathrm{~min}^{-1} \cdot \mu \mathrm{Ci}^{-2} \\
t^{*} & =1000 \mathrm{s.}
\end{array}
$$

Then with $\left(M^{\mathrm{air}} / M^{\mathrm{K} \mathrm{r} 85}\right)^{1 / 2}=1 / 1.71$, one obtains

$$
\begin{aligned}
L_{\mathrm{Kr}^{85}} \text { reject } & =5 \times 10^{-8}\left(\frac{M^{a}}{M^{\mathrm{Kr}}}\right)^{1 / 2} \\
& =2.92 \times 10^{-8} \mathrm{~atm} \cdot \mathrm{cm}^{3} / \mathrm{s}
\end{aligned}
$$

whence

$$
R_{r}{ }^{*}=2.92 \times 10^{-6} \mathrm{~s}^{-1}
$$

so

$$
R^{*} /\left(P_{0} V P_{E}{ }^{*} S K\right)=E^{*}=2.5 \times 10^{-2} \mathrm{~atm}^{-1} .
$$

As shown in Fig. 4 a line is erected at $R_{r}=2.94 \times 10^{-6}$ $\mathrm{s}^{-1}$, and a horizontal test line is placed at $E^{*}=2.5 \times 10^{-2}$. At the intersection, $T=2.4 \mathrm{~h}$. At the intersection with the $t=1000 \mathrm{~s}$ segment, $R_{r}{ }^{*} \max =3.7 \times 10^{-3} \mathrm{~s}^{-1}$ such that $L_{\mathrm{air}}=6.3 \times 10^{-5} \mathrm{~atm} \cdot \mathrm{cm}^{3} / \mathrm{s}$. Note that leak rates are for $\mathrm{Kr}^{85}$ and hence $R_{r}$ values are smaller than for helium for a given air leak.

Were the reject limit lowered to an $L_{\text {air }}$ of $1 \times 10^{-8}$ atm $\cdot \mathrm{cm}^{3} / \mathrm{s}, R_{r}{ }^{*}$ would be $5.88 \times 10^{-7} \mathrm{~s}^{-1}$ so that $T$ is $11.8 \mathrm{~h}$; that is, the pressurization time changes linearly with $R_{r}$, as evidenced in Fig. 4 and in (20) for the straight line projection region of $E(T)$.

2) Repetitive Measurement: An initial count rate made on the specimen relates to the quantity of $\mathrm{Kr}^{85}$ already within by (17). After a lapsed time $t^{\prime \prime}$ the specimen is pressurized. The interior pressure just before this is equivalent to that expressed in (12) or

$$
P^{\prime}=\frac{R_{1}^{*}}{V \cdot A \cdot K} \exp \left(-R_{r}{ }^{*} t^{\prime \prime}\right)
$$

The final pressure after processing is as in (4), and the final count rate is again determined by (17) as

$$
\begin{aligned}
& \frac{R_{2}^{*}-R_{1}{ }^{*} \exp \left(-R_{r} t^{\prime \prime}\right)\left[\exp \left(-R_{r}{ }^{*} t_{2}\right)-P_{0} E_{2}{ }^{*}\right]}{P_{0} V \cdot P_{E}{ }^{*}} \\
& \quad=S K \cdot E_{2}{ }^{*}
\end{aligned}
$$

which is of the same form as (14). In the small leak range where $\exp \left(-R_{r}{ }^{*} t^{\prime \prime}\right)$ and $\exp \left(-R_{r}{ }^{*} t_{2}\right)$ are $\sim 1$, and as $E_{2} * \ll 1$, the initial count rate is a direct correction or

$$
\frac{R_{2}^{*}-R_{1}^{*}}{P_{0} V \cdot P_{E}{ }^{*}}=S K \cdot E_{2}^{*} \text {. }
$$




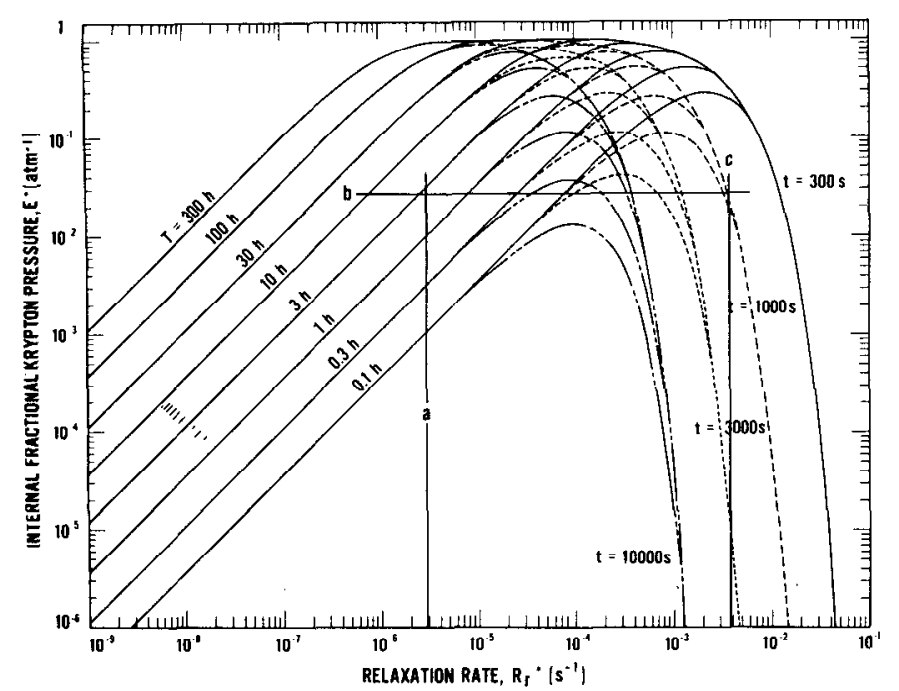

Fig. 4. Test line and example of test range for radioisotope method. Reject leak size of $5 \times 10^{-8} \mathrm{~atm} \cdot \mathrm{cm}^{3} / \mathrm{s}$ of air with $V=0.01 \mathrm{~cm}^{3}$ $P_{E}{ }^{*}=5 \mathrm{~atm}$ of $\mathrm{Kr}-\mathrm{N}_{2}$ mixture, $S=200 \mu \mathrm{Ci} / \mathrm{atm} \cdot \mathrm{cm}^{3}, K=10^{4}$ min-1. $\mu \mathrm{Ci}^{-1}$, and a minimum detectable count rate of 2500 $\min ^{-1}$ for a signal-to-noise ratio of 5. Line (a) represents $R_{r}{ }^{*}$ of $2.94 \times 10^{-6} \mathrm{~s}^{-1}$ for $\mathrm{Kr}^{85} . R^{*} /\left(P_{0} V P_{E^{*}} S K\right)=E^{*}=2.5 \times 10^{-2}$ $\mathrm{atm}^{-1}$ is test line (b). The intersection of (a) and (b) sets $T=2.4 \mathrm{~h}$. At the intersection of (b) and the $t=1000 \mathrm{~s}$, line (c) represents $R_{r}{ }^{*}=3.7 \times 10^{-3} \mathrm{~s}^{-1}$ which leads to $L_{\mathrm{air}}=6.3 \times 10^{-5} \mathrm{~atm} \cdot \mathrm{cm}^{3} / \mathrm{s}$.

In the large leak range where $\exp \left(-R_{r} * t^{\prime \prime}\right)<1, \exp \left(-R_{r}{ }^{*} t_{2}\right) \rightarrow$ 0 , and $E_{2} \ll 1$, the prereading is not significant. In the intermediate range, (22) is solved by iteration just as for the helium leak detector with the exception of the horizontal test lines and $R_{r} *$ values for $\mathrm{Kr}^{85}$ rather than for helium.

\section{COMPARISON OF HELIUM AND RADIOISOTOPE METHODS}

Within the molecular flow regime test results obtained with the two methods can be compared with the use of characteristic leak-test curves. The capability of the helium leak detector method is oriented toward the larger leak sizes and smaller volumes, for the pressurization time increases rapidly along the helium test line as the relaxation rate diminishes. The radioisotope method requires a longer pressurization time with intermediate leak sizes because the leak rate for $\mathrm{Kr}^{85}$ is some 4.6 times smaller than that for $\mathrm{He}$ in the same leak channel; however the pressurization time increases but linearly as the relaxation rate diminishes. The radioisotope test line tends to lie in the nonlinear portion of the characteristics, particularly at the large leak end, but in this range the $45^{\circ}$ slope of the helium leak detector method tends to both the smaller values for $E$ and the larger values of $R_{r}$ where straight line segments give reasonable approximation. However in the conversion to air leak rates, both methods produce the same approximate upper limit when the same dwell times are used. The radioisotope method does allow shorter dwell times in use and therefore can in principle extend to a larger upper limit of leak size. The short dwell time obtainable with the radioisotope method also allows two cycle testing for extension to gross leak sizes [13].
TABLE I

COMPARATIVE VALUES FOR HELIUM LEAK DETECTOR AND RADIOISOTOPE TESTING OF HERMETIC PACKAGES!

\begin{tabular}{cccccc}
\hline \hline $\begin{array}{c}V \\
\left(\mathrm{~cm}^{3}\right)\end{array}$ & $\begin{array}{c}L_{\mathrm{air}} \\
\left(\mathrm{atm} \cdot \mathrm{cm}^{3} / \mathrm{s}\right)\end{array}$ & $\begin{array}{c}R_{r} \\
\left(\mathrm{~s}^{-1}\right)\end{array}$ & \multicolumn{1}{c}{$T^{*}$} & \multicolumn{1}{c}{$T / T^{*}$} & $n^{* / n}$ \\
\hline 0.01 & $10^{-6}$ & $2.7 \times 10^{-4}$ & $255 \mathrm{~s}$ & 0.0016 & \\
& $10^{-7}$ & $2.7 \times 10^{-5}$ & $0.71 \mathrm{~h}$ & 0.016 & \\
& $10^{-8}$ & $2.7 \times 10^{-6}$ & $7.1 \mathrm{~h}$ & 0.16 & 0.16 \\
10 & $10^{-6}$ & $2.7 \times 10^{-7}$ & $255 \mathrm{~s}$ & 1.6 & \\
& $10^{-7}$ & $2.7 \times 10^{-8}$ & $0.71 \mathrm{~h} 16$ & \\
& $10^{-8}$ & $2.7 \times 10^{-9}$ & $7.1 \mathrm{~h} 161$ & 161 \\
\hline
\end{tabular}

1 Derived from (23). Pressurization times $T / T^{*}$ for equal signal-tobackground ratios, and signal-to-background ratios $n^{*} / n$ for equal pressurization times. $S=200 \mu \mathrm{Ci} / \mathrm{atm} \cdot \mathrm{cm}^{3}, K=10^{4} \mathrm{~min}^{-1} \mu \mathrm{Ci}-1, R_{0}=$ $5 \times 10^{-10} \mathrm{~atm} \cdot \mathrm{cm}^{3} / \mathrm{s}$ helium, $R_{0}{ }^{*}=500 \mathrm{~min}^{-1}$.

A direct numerical comparison can also be obtained for the fine leaks by using (19) for the radioisotope method, (11) for the helium method, the approximation of (7), and the detector outputs in terms of signal-to-background, for the same pressurization value, as

$$
\frac{n^{*} R_{0}{ }^{*}}{n R_{0}}=\frac{S K}{4.61 R_{r}} \cdot \frac{T^{*}}{T}
$$

where the ratio $R_{r} *$ to $R_{r}$ is equal to the ratio of the standard leak rates for a given leak size which is $\left(M^{\mathrm{He}} / M^{\mathrm{Kr}}\right)^{1 / 2}$ or $1 / 4.61, n$ is the signal-to-background ratio, and $R_{0}$ is the threshold signal. Some results are listed in Table $\mathbf{I}$. Note that the radioisotope pressurization times are independent of volume per (20). A comparison of signal-to-background ratios is listed in the last column for equal pressurization times.

\section{SUMMARY}

The molecular flow formulation for the back pressurization method of hermetic testing has been condensed into a normalized set of characteristic curves from which specific solutions can be obtained by means of a test line. The characteristics are appropriate for both the helium leak detector and and radioisotope methods of test, while the form of the test line distinguishes between two methods. Minimum detectable and maximum detectable leak sizes can be determined simultaneously from the one set, and the selection of suitable test conditions is facilitated. Procedures are also derived for applying corrections due to prior testing or prefill.

\section{REFERENCES}

[1] S. Ruthberg. in Semiconductor Measurement Technology: ARPA/ NBS Workshop II. Hermeticity Testing for Integrated Circuits, H. A. Schafft, Ed., NBS Special Publication 400-9, pp. 27-32, Dec. 1974. (Available from U.S. Govt. Printing Office, SD no. C13.10:400-9.)

[2] S. Dushman and J. M. Lafferty, Scientific Foundations of Vacuum Technology, 2nd ed., section 2.5, New York: Wiley, 1962, p. 104.

[3] D. A. Howl and C. A. Mann, "The back-pressurization technique of leak testing,"'Vacuum, vol. 15, pp. 347-352, 1965.

[4] S. B. Banks, R. E. McCullough, and E. G. Roberts, "Investigation 
of microcircuit seal testing, " Air Force Systems Command. Rome Air Development Center, Tech. Rep. RADĆ-TR-75-89, Apr. 1975.

15] W. E. Briggs and S. G. Burnett, "Helium mass spectrometer leak testing of pressure--"bombed" sealed enclosures," Varian Vacuum Division/NRC Operation, Newton Highlands, MA, Oct. 1, 1968.

[6] Standard Recommended Practices for Determining Hermeticity of Electron Devices with a Helium Mass Spectrometer Leak Detector, ASTM Designation F134, 1979 Annual Book of ASTM Standards, p. 545-552.

[7] B. Cassen and D. Burnham, "A method of leak testing hermetically sealed components utilizing radioactive gas," Int. J. Appl. Radiation and Isotopes, vol. 9, pp. 54-59, 1960.

[8] Method 1014.2-Seal, Military Standard 883B, in Test Methods and Procedures for Microelectronics, Aug. 31, 1977. (Available from U.S. Naval Publications and Forms Center, 5801 Tabor Ave., Philadelphia, PA 19120.)

[9] S. Dushman and J. M. Lafferty, Scientific Foundations of Vacuum Technology. 2nd ed., section 2.2, New York: Wiley, 1962, p. 81.

[10] Glossary of Terms Used in Vacuum Technology, Committee on Standards, American Vacuum Society Inc. New York: Pergamon, 1958, Definitions No. 20 and 236.

[11] S. Dushman and J. M. Lafferty, Scientific Foundations of Vacuum Technology, 2nd ed., New York: Wiley, 1962, p. 16.

[12] AVS Helium Mass Spectrometer Leak-Detector Calibration (2.11963), J. Vac. Sci. Technol., vol. 3, pp. 229-235, 1966.

[13] S. Ruthberg, G. R. Neff, and B. D. Martin, "Radioisotope hermetic test precision," in Proc. 1977 Int. Microelectronics Symposïum, Baltimore, MD, Oct. 24-26, 1977, pp. 131-137.

\title{
High Density Multiwire Circuits Using Thinner Wires
}

\author{
ETSUJI SUGITA, OSAMU IBARAGI, AND SHIGEHARU MOMOI
}

\begin{abstract}
High density Multiwire circuit technology which can lay two insulated wires between throughholes on $2.5 \mathrm{~mm}$ centers has been developed. To reduce crosstalk smaller diameter wires were adopted instead of conventional $0.16 \mathrm{~mm}$ diameter wires. Wire breakage and wiring mistakes, which occur in high density wiring, can result from improper wire tacking. A newly developed tackless wiring technique can reduce these problems. The new technology has been applied to backplane wiring boards in an electronic switching system.
\end{abstract}

\section{INTRODUCTION}

$\mathbf{T}$ THE MAIN differences between Multiwire ${ }^{\circledR}$ circuits [1] and printed circuits are that in Multiwire circuits a) insulated wires are embedded into the adhesives by a numerically controlled machine and b) cross-section of a wire is directly connected to a plated throughhole. Therefore Multiwire technology simplifies wiring design while maintaining the possibility of high interconnection density. Due to these features Multiwire technology has attracted attention as an alternative to the traditional multilayer printed circuits [2] or WireWrap $^{\circledR}$ [3] for backplane wiring in electronic equipments.

Conventional Multiwire circuits have employed $0.16 \mathrm{~mm}$ diameter wires and have permitted only one wire between throughholes on $2.5 \mathrm{~mm}$ centers. In the application to backplane wiring it is necessary to improve the ability for wire accommodation. High density wiring will be effective for achievment of this capability. However if two $0.16 \mathrm{~mm}$ wires are laid, many wiring mistakes will occur and crosstalk will increase. To solve these problems high density wiring using thinner wires was studied.

Manuscript received June 12, 1980; revised December 31, 1980.

The authors are with the Physical Design Section, Electronic Equipment Development Division, Masashino Electrical Communication Laboratory, NTT, Musashino-shi, Tokyo 180, Japan.

${ }^{8}$ Multiwire is a registered trademark of Kollmorgen Corporation and Wire-Wrap is a registered trademark of Gardner-Denver.
This paper describes the crosstalk problem, a new wiring technique for high density Multiwire circuits, and backplane wiring boards produced by high density Multiwire technology.

\section{CROSSTALK}

Although crosstalk measurements on multilayer printed circuits are reported by [4] etc., measurements on high density Multiwire circuits have not yet been reported. In the following paper crosstalk is described, and the effectiveness of thinner wires in reducing crosstalk is shown.

Crosstalk equations for pulsed signals between parallel and matched microstrip lines are given by (4) and (5).

$$
\begin{aligned}
V_{N} & =\frac{1+\alpha}{4} \cdot \frac{L_{m}}{L} \cdot V_{D}, \quad t_{r} \leqq 2 \tau l \\
& =\frac{1+\alpha}{4} \cdot \frac{L_{m}}{L} \cdot \frac{2 \tau l}{t_{r}} \cdot V_{D}, \quad t_{r}>2 \tau l \\
V_{F} & =-\frac{1-\alpha}{4} \cdot \frac{L_{m}}{L} \cdot \frac{2 \tau l}{t_{r}} \cdot V_{D}
\end{aligned}
$$

where $V_{N}$ is near-end crosstalk voltage, $V_{F}$ is farend crosstalk voltage, $V_{D}$ is drive voltage, $\tau$ is propagation delay, $l$ is parallel length, $t_{r}$ is rise time, $\alpha$ is $\left(C_{m} / C\right) /\left(L_{m} / L\right), C_{m}$ is mutual capacitance/unit length, $C$ is self-capacitance/unit length, $L_{m}$ is mutual inductance/unit length, and $L$ is self-inductance/unit length.

As Multiwire circuits have the structure shown in Fig. 1, their $L$ and $L_{m}$ are given by the following forms [6], respectively.

$$
L=\frac{\mu}{2 \pi} \ln \frac{2 h}{a}
$$

